\title{
Adaptive Hierarchical-Concurrent Multiscale Modeling of Ductile Failure in Heterogeneous Metallic Materials
}

\author{
SOMNATH GHOSH ${ }^{1,2}$ \\ 1.-Johns Hopkins University, Baltimore, MD 21218, USA. 2.-e-mail: sghosh20@jhu.edu
}

\begin{abstract}
This article addresses two topics on multiscale modeling of heterogeneous metals and alloys. The first topic focuses on developing an adaptive hierarchical-concurrent multilevel modeling framework for ductile fracture. The microstructure of aluminum alloys, for example, is characterized by a dispersion of heterogeneities such as silicon and intermetallics in a ductile aluminum matrix. The multilevel model invokes two-way coupling, viz. hierarchical models for homogenized constitutive modeling and concurrent models with scale transition in regions of localization and damage. Adaptivity is necessary for evolving microstructural deformation and damage. A macroscopic analysis in regions homogeneity incorporates homogenization-based continuum plasticity-damage models. A microscopic analysis using locally enhanced-Voronoi cell finite-element method is required for regions of high macroscopic gradients caused by underlying localized plasticity and damage. Coupled macroscopic and microscopic analysis is conducted concurrently. Physics-based level change criteria are developed to improve accuracy and efficiency. The second topic discusses a nested dual-stage homogenization method for microstructure-based homogenized continuum plasticity models for cast aluminum alloys with large secondary dendrite arm spacing. Two distinct statistically equivalent representative volume elements are identified and used in the asymptotic expansion-based homogenization and self-consistent homogenization processes, respectively. The two-stage homogenization enables an evaluation of the overall homogenized model of a cast alloy from limited experimental data, as well as material properties of constituents like interdendritic phase and pure aluminum matrix.
\end{abstract}

\section{INTRODUCTION}

Heterogeneous metallic materials like cast aluminum alloys, containing silicon and intermetallic inclusions in a dendritic structure, are widely used in a variety of engineering applications such as in automotive and aerospace structures. While microstructural heterogeneities may result in higher strength in some cases, failure properties like ductility and strain to failure are generally affected adversely by particulate fragmentation and matrix cracking. Ductile failure generally initiates with particulate fragmentation. Large plastic strains in their vicinity lead to void nucleation in the matrix. The damage subsequently propagates with void growth and localizes in bands of intense plastic deformation between particulates, until void coalescence in the matrix leads to catastrophic failure. Experimental studies, e.g., in Ref. 1, have shown strong connections between morphological variations and microstructural damage nucleation and growth. Damage initiation is controlled by the shape of particulates, whereas the rate of damage evolution at higher strains is controlled by the level of clustering. This makes predicting ductility and strain to failure quite challenging. Modeling the ductile failure process using exclusively macroscopic models will lead to large predictive inaccuracies due to the strong dependence of deformation and failure on the microstructure. Pure micromechanical analysis of the entire structure, on the other hand, is computationally intractable due to the large number of heterogeneities in the underlying microstructure. The need for multiscale modeling is clearly realized for these problems. 
Different classes of multiscale models have been developed in the literature. These multiscale models have been classified as hierarchical, concurrent, or a combination of both. The hierarchical multiscale methods involve bottom-up homogenization (oneway scale coupling) to develop response models for the higher scales. Hierarchical methods using homogenization techniques have been developed, e.g., in Refs. 2-11, based on the asymptotic expansion theory of homogenization. Higher-order homogenization methods have been developed, e.g., in Refs. 12-15, for incorporating higher-order kinematics. Concurrent multiscale methods that involve top-down analysis and introduce coupled analysis of the substructured computational domain consisting of regions of macroscopic analysis with homogenized material properties and embedded local regions of detailed micromechanical modeling have been developed, e.g., in Ref. 16.

By combining the two methods in an integrated framework, Ghosh and co-workers have developed adaptive, hierarchical-concurrent image-based multiscale models for linear elastic composites in Refs. 17 and 18, elastic-plastic composite materials undergoing microstructural damage by inclusion cracking in Ref. 19, composites with micromechanical debonding in Ref. 20, and rate-dependent ductile fracture in heterogeneous metals and alloys with non-uniform microstructures in Refs. 21-23. Detailed developments of the multiscale analysis framework using the Voronoi cell finite-element method (VCFEM) for micromechanical analyses have been presented in Ref 11. Three major ingredients constitute the image-based multiscale analysis framework. They are (I) multiscale image analysis and characterization, (II) micromechanical analysis of extended microstructural regions, and (III) adaptive concurrent multiscale analysis incorporating both bottom-up and top-down modeling. Powerful methods of image-reconstruction, multiscale image characterization and domain partitioning have been developed in Refs. 24 and 25 as preprocessors to image-based multiscale modeling. These modules are necessary for determining microstructural representative volume elements (RVEs), as well as for identifying regions where homogenization breaks down. The two-way coupled multiscale framework invokes a macroscopic analysis with hierarchically constructed homogenized constitutive models in less critical regions of low deformation or stress gradients to enhance efficiency of the computational analysis. In critical regions of localization, damage, and failure, the model cascades down to the scale of the embedded microstructure with explicit representation of morphology and deformation mechanisms. The topdown coupling is achieved in a concurrent framework. Adaptivity, which implies computational model-determined selection of regions that require different resolutions, is a necessary feature in the computational model. Regions of microstructure embedding cannot be determined a priori for problems with evolving microstructures and damage mechanisms. The adaptive substructuring should be guided by physical considerations to minimize modeling errors.

This article will address two topics on multiscale modeling of heterogeneous aluminum alloys. The first topic focuses on developing the adaptive hierarchical-concurrent multilevel modeling framework for ductile fracture. It incorporates mechanisms of damage nucleation by particle cracking, with subsequent void growth and void coalescence. Hierarchical modeling is invoked for developing a homogenization-based continuum rate-dependent plasticity-damage (HCPD) model for regions exhibiting macroscopic homogeneity. The model zooms down and introduces micromechanical modeling to explicitly depict particle cracking and void growth in regions of high macroscopic gradients. Computational adaptivity is necessary to account for continuous changes in the model as a consequence of evolving microstructural deformation and damage. Physics-based level change criteria control transcending scales in the model.

The second topic discusses a nested dual-stage homogenization method for microstructure-based homogenized continuum plasticity (HCP) models of cast aluminum alloys with large secondary dendrite arm spacing (SDAS). Microstructures of these alloys are characterized by inhomogeneous distribution of inclusions along the dendrite cell boundaries (see Fig. 10). Traditional single-step homogenization methods are not suitable for these microstructures due to the size requirements on RVEs. To circumvent this limitation, two distinct statistically equivalent RVEs are identified, corresponding to the inherent scales of inhomogeneity in the microstructure. Homogenization is performed in multiple stages for each RVE identified. The nested twostage homogenization enables evaluation of the overall homogenized model of the cast alloy from limited experimental data, as well as material properties of constituents like interdendritic phase and pure aluminum matrix.

\section{THE HIERARCHICAL-CONCURRENT MULTISCALE MODELING FRAMEWORK}

The multilevel framework for multiscale analysis adaptively decomposes the heterogeneous computational domain $\Omega_{\text {het }}$ into a set of nonintersecting subdomains, denoted by level 0 , level 1 , level 2 , and level tr, i.e., $\Omega_{\text {het }}=\Omega_{10} \cup \Omega_{11} \cup \Omega_{12} \cup \Omega_{\text {tr }}$. The schematic of this hierarchy and domain decomposition is shown in Fig. 1. The characteristics of the levels may be delineated as follows:

- Level 0 corresponds to subdomain $\Omega_{10}$ of pure macroscopic analysis.

- Level 1 corresponds to a swing subdomain $\Omega_{11}$ that assesses the appropriateness of macroscopic analysis over pure micromechanical analysis. 
- Level 2 corresponds to subdomain $\Omega_{12}$ of pure micromechanical analysis of extended microstructural images with no periodicity constraints.

- Level tr corresponds to transition subdomain $\Omega_{\text {tr }}$ for interconnecting regions of homogeneous macroscopic analysis with those of heterogeneous microscopic analyses.Concurrent multiscale analysis requires that all subdomains $\Omega_{10}, \Omega_{11}, \Omega_{12}$, and $\Omega_{\text {tr }}$ be coupled and solved simultaneously as discussed in Refs. 11 and 22. The levels are defined next.

\section{Computational Subdomain Level $0\left(\Omega_{10}\right)$}

The level 0 computational subdomain $\left(\Omega_{10}\right)$ assumes homogeneous macroscopic deformation and uses constitutive models that are obtained from hierarchical multiscaling analysis. Hierarchical methods such as $\mathrm{FE}^{2}$ methods ${ }^{7}$ solve the micromechanical RVE or unit cell problem at every integration point of the finite-element mesh in each time-increment to obtain properties for macroscopic analysis. To overcome the limitations of prohibitive computational costs, Ghosh et al. ${ }^{20,26}$ have developed homogenized elastic-plastic-damage constitutive laws by homogenizing the microscopic RVE response. The constitutive models account for the effect of morphological features and evolving microstructural mechanisms through evolving, anisotropic homogenized parameters. These reduced-order constitutive models are computationally very efficient as they do not have to account for the details of microstructural morphology or solve the micromechanical RVE problem in every step of an incremental process.

For macroscopic analysis of ductile failure in porous viscoplastic materials with brittle inclusions, Ghosh et al. have developed rate dependent/independent HCPD models in Refs. 26-28. A sequence of micromechanical analyses of the RVE (e.g., in Fig. 2b) under different load histories are conducted for this development. Parameters in the HCPD models are calibrated to represent the effect of morphology, as well as evolving microstructural mechanisms. The anisotropic HCPD models have the framework of the Gurson-Tvergaard-Needleman (GTN) models developed in Refs. 29-31. An anisotropic plastic flow potential is introduced in an evolving material principal coordinate system, for which the parameters evolve as functions of plastic work. Important steps in the development of the HCPD model are discussed next.

\section{Identification of the Statistically Equivalent Repre- sentative Volume Element (SERVE)}

A SERVE is the smallest, location-independent, microstructural domain necessary for micromechanical analysis leading to homogenization. The effective material properties for the SERVE should equal those for the entire microstructure. Methods of SERVE identification from real micrographs have been developed in Refs. 26-28. In this work, the multivariate marked correlation function $M(r)$, which relates any geometric or response field variable with the microstructural morphology, is used to establish the SERVE. In Ref. 26, $M(r)$ has been calculated with the micromechanical plastic work $W_{\mathrm{p}}$ as the mark. As shown in Fig. 2a, $M(r)$ stabilizes to near-unity values at a characteristic radius of convergence $r_{0}$, signifying a correlation length. For $r>r_{0}, M(r) \approx 1$ and the local morphology ceases to have any significant influence on the field variables beyond this characteristic radial distance. The SERVE size is estimated as $L_{\mathrm{RVE}} \approx 2 \times r_{0}$, where $r_{0}$ corresponds to the local correlation length. For the cast aluminum alloy W319 microstructure in Fig. 2c the SERVE size has been established in Ref. 28 as $L_{\mathrm{RVE}}=48 \mu \mathrm{m}$.

\section{Homogenization-Based Continuum Plasticity- Damage Constitutive Relations}

The macroscopic HCPD model is established from a micromechanical analysis of the SERVE using the Hill-Mandel condition of homogeneity. ${ }^{32}$ This condition states that for large differences in microscopic
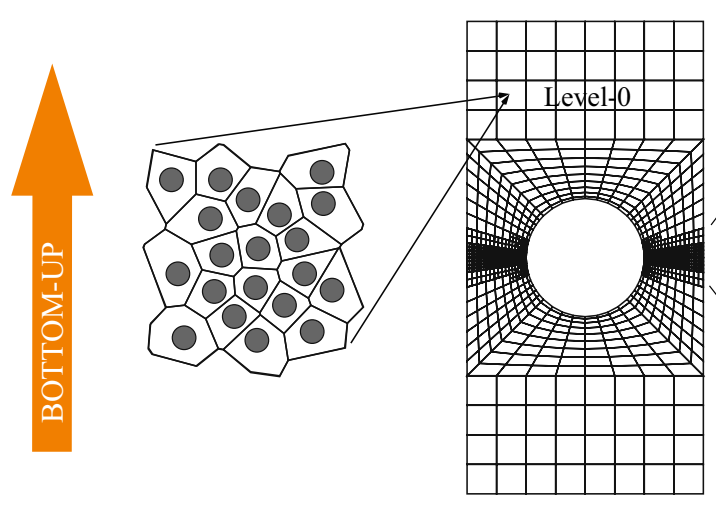

(a)
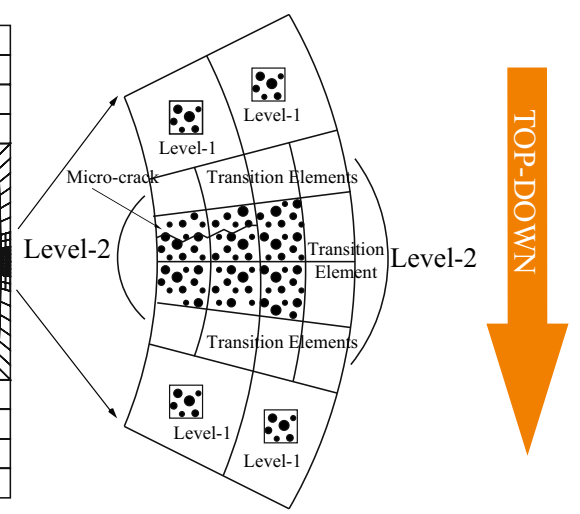

(c)

Fig. 1. An adaptive, two-way coupled multiscale analysis model: (a) RVE for constructing homogenized continuum models for level 0 analysis, (b) a level 0 model with adaptive zoom-in, and (c) zoomed-in level 1, level 2, and transition subdomains. 


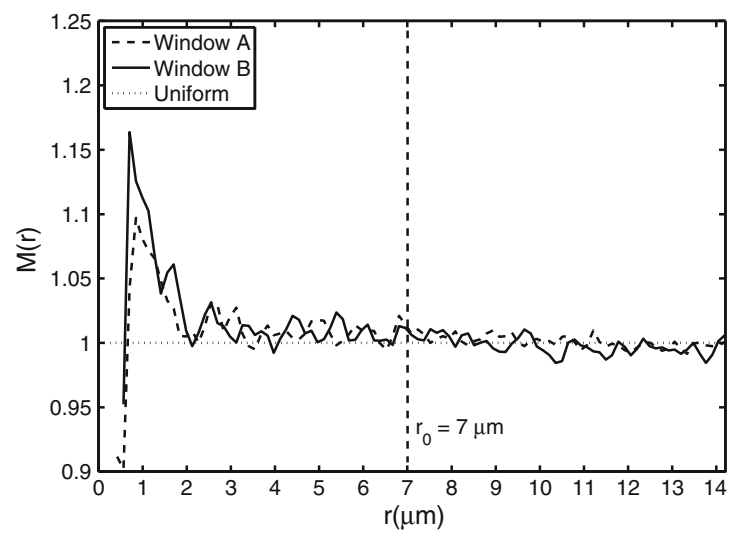

(a)

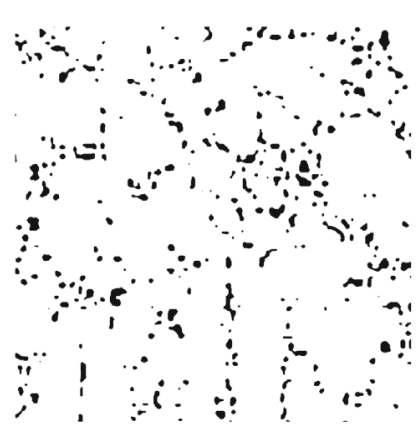

(b)

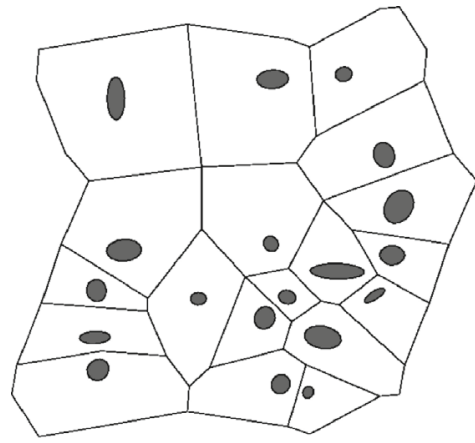

(c)

Fig. 2. (a) Marked correlation function for a cast aluminum alloy micrograph showing convergence to the SERVE size, (b) micrograph of a cast aluminum alloy W319 $(192 \mu \mathrm{m} \times 192 \mu \mathrm{m})$ showing dendritic regions, and (c) typical SERVE of the microstructure in (b) obtained for a characteristic size of $48 \mu \mathrm{m}$ (Micrograph: Courtesy Ford Research Laboratory).

and macroscopic length scales, the volume-averaged strain energy is obtained as the product of the volume-averaged stresses and strains in the RVE, i.e.,

$$
\int_{\Omega_{\mathrm{RVE}}} \sigma_{i j}^{*} \epsilon_{i j}^{*} d \Omega=\left\langle\sigma_{i j}^{*} \epsilon_{i j}^{*}\right\rangle=\left\langle\sigma_{i j}^{*}\right\rangle\left\langle\epsilon_{i j}^{*}\right\rangle
$$

where $\sigma_{i j}^{*}$ and $\epsilon_{i j}^{*}$ are the equilibrated stress and kinematically admissible strain fields in the microstructure, respectively; $\Omega_{\mathrm{RVE}}$ is the microstructural SERVE volume; and $\langle\cdot\rangle$ is the Macaulay bracket. Micromechanical analysis in the present work is conducted by the locally enhanced Voronoi cell finite-element method or LE-VCFEM ${ }^{11}$ that is summarized in the section "Computational Subdomain Level $2\left(\Omega_{12}\right)$ of Micromechanical Analyses."

The rate-dependent HCPD model for porous viscoplastic materials containing brittle inclusions in Refs. 27 and 28 is based on an anisotropic GTN model framework. The homogenized Cauchy stress rate is related to the elastic strain-rate tensor as:

$$
\dot{\Sigma}=\overline{\mathbf{C}}^{\mathbf{e}}: \dot{\mathbf{e}}^{\mathbf{e}}=\overline{\mathbf{C}}^{\mathbf{e}}:\left(\dot{\mathbf{e}}-\dot{\mathbf{e}}^{\mathbf{p}}\right)
$$

where $\overline{\mathbf{C}}^{\mathbf{e}}$ is a homogenized fourth-order anisotropic elasticity tensor. Elastic anisotropy is due to the distribution of heterogeneities in the microstructure. The total homogenized strain rate is additively decomposed into homogenized elastic and viscoplastic parts as $\dot{\mathbf{e}}=\dot{\mathbf{e}}^{\mathbf{e}}+\dot{\mathbf{e}}^{\mathbf{p}} . \bar{\phi}$ is an effective flow potential, representing the stress-space loading surface for the homogenized material with underlying matrix containing particulates and evolving voids. Following the structure of GTN models in Refs. 29 and $31, \bar{\phi}$ is expressed in terms of the hydrostatic $\left(\Sigma^{\text {hyd }}\right)$ and deviatoric $\left(\Sigma_{\text {eq }}\right)$ parts of the homogenized Cauchy stress tensor as:

$$
\bar{\phi}=\left(\frac{\Sigma_{\mathrm{eq}}}{\bar{\sigma}_{\mathrm{c}}}\right)^{2}+2 Q_{1} \bar{f} \cosh \left(\frac{3 Q_{2}}{2} \frac{\Sigma^{\mathrm{hyd}}}{\bar{\sigma}_{\mathrm{c}}}\right)-1-\left(Q_{1} \bar{f}\right)^{2}=0
$$

where $\bar{f}$ is the homogenized void volume fraction. The parameters $Q_{1}$ and $Q_{2}$ are introduced in Ref. 26 to capture the effect of void interaction. The flow potential in Eq. 3 exhibits anisotropy emanating from two sources, viz. (I) dispersion of brittle inclusions in the matrix and (II) evolution of damage (voids) in the microstructure. Anisotropy is accounted for through a homogenized equivalent stress $\Sigma_{\text {eq }}$ following the anisotropic yield function in Ref. 33. Under plane strain condition, this is expressed as:

$$
\Sigma_{\text {eq }}^{2}=F\left(\Sigma_{y y}-\Sigma_{z z}\right)^{2}+G\left(\Sigma_{z z}-\Sigma_{x x}\right)^{2}+H\left(\Sigma_{x x}-\Sigma_{y y}\right)^{2}+C \Sigma_{x y}^{2}
$$

All variables in Eqs. 3 and 4 are expressed in an evolving, material-damage principal (MDP) coordinate system. The anisotropy parameters $F, G, H$ and $C$ have been found to be functions of the evolving plastic work $W_{p}$ in the SERVE in Ref. 26. In Eq. $3, \bar{\sigma}_{c}$ corresponds to the averaged stress in the material consisting of matrix and inclusions, but without voids. The corresponding over-stress $F$ in the viscoplasticity flow rule ${ }^{34}$ is expressed as a measure of the excess stress over the rate-independent local yield strength, as:

$$
\bar{F}=\bar{\sigma}_{\mathrm{c}}-Y_{\mathrm{f}}\left(W_{\mathrm{p}}\right)
$$

where $Y_{f}$ is the rate-independent homogenized yield strength of the underlying heterogeneous material without voids, which depends on the plastic work $W_{\mathrm{p}}$. The homogenized viscoplastic strain-rate tensor, normal to the loading surface $\bar{\phi}(\bar{F})$ in the stress space, is derived as:

$$
\dot{\mathbf{e}}^{\mathbf{p}}=\dot{\Lambda} \frac{\partial \bar{\phi}}{\partial \Sigma}=\frac{(1-\bar{f}) \overline{\sigma_{c}}}{\Sigma: \frac{\partial \bar{\phi}}{\partial \Sigma}} \Gamma_{0} \Phi(\bar{F}) \frac{\partial \bar{\phi}}{\partial \Sigma}
$$


The homogenized viscoplastic multiplier ${ }^{\circ}$ is obtained by enforcing the Hill-Mandel condition. ${ }^{32}$ To is a temperature-dependent viscosity coefficient and the function is assumed to be $(\bar{F})=\langle\bar{F}\rangle^{P}$. Finally, the evolution equations for the homogenized plastic work $W_{\mathrm{p}}$, yield stress $Y_{\mathrm{f}}$, and void volume fraction $\bar{f}$ are expressed as:

$$
\dot{W}_{\mathrm{p}}=\Sigma: \dot{\mathbf{e}}^{\mathrm{p}}, \dot{Y}_{\mathrm{f}}=\frac{\partial Y_{\mathrm{f}}}{\partial W_{\mathrm{p}}} \dot{W}_{\mathrm{p}}, \quad \dot{\bar{f}}=\dot{\bar{f}}_{\text {growth }}+\dot{\bar{f}}_{\text {nucleation }}
$$

where $\dot{\bar{f}}_{\text {growth }}=(1-\bar{f}) \dot{e}_{k k}^{p}$. The homogenized void nucleation rate $f_{\text {nucleation }}$ follows directly from the inclusion cracking statistics in the underlying microstructural SERVE.

A strain-based homogenized void nucleation model is developed in Ref. 28, accounting for the effects of the underlying microstructural morphology and rate dependency. It invokes the Weibull statistics-based probability function that is used to initiate inclusion cracking in Eq. 12. The macroscopic nucleation probability function $\bar{P}_{\text {frag }}$ is written in terms of the homogenized strain tensor and its rate, as well as the particulate size $v$ as:

$$
\bar{P}_{\text {frag }}(v, \hat{e}, \dot{\hat{e}})=1-\exp \left[-\frac{v}{v_{0}}\left(\frac{\hat{e}}{e_{0}(\dot{\hat{e}})}\right)^{m(\dot{\hat{e}})}\right]
$$

where $e_{0}$ and $m$ are the Weibull parameters and $v_{0}$ is a reference volume. The Weibull parameters are determined to be functions of an effective strain rate $\dot{\hat{e}}$, where $\hat{e}=\left\langle A\left(\theta_{\mathrm{p}}\right) e_{1}+B\left(\theta_{\mathrm{p}}\right) e_{2}+C\left(\theta_{\mathrm{p}}\right) e_{3}\right\rangle$ is an effective strain measure in terms of the macroscopic principal strains $e_{i}$. The coefficients $A, B$, and $C$ are functions of direction of the maximum principal strain. The area fraction of cracked inclusions for a given strain rate is expressed in terms of the probability density function of the inclusion size $p(v)$ and the probability of inclusion fragmentation $\bar{P}_{\text {frag }}(v, \hat{e}, \dot{\hat{e}})$. For a discrete size distribution in a finite-sized SERVE, the area fraction is expressed as:

$$
\rho_{c}(\hat{e}, \dot{\hat{e}})=\sum_{i=1}^{N} \frac{v_{i}}{v_{0}} p\left(v_{i}\right)\left(1-\exp \left[-\frac{v_{i}}{v_{0}}\left(\frac{\hat{e}}{e_{0}(\dot{\hat{e}})}\right)^{m(\hat{\hat{e}})}\right]\right)
$$

$N$ is the number of discrete inclusion sizes $v_{i}$ in the probability density function $p(v)=\sum_{i=1}^{N} \delta\left(v-v_{i}\right) p\left(v_{i}\right)$, where $\delta\left(v-v_{i}\right)$ is the Dirac delta function. To account for variations in strain rates, the rate of evolution of the area fraction of cracked inclusions $\rho$ is assumed to be governed by the relation:

$$
\dot{\rho}=\tilde{k}^{\star} \frac{d \rho_{\mathrm{c}}(\hat{e}, \dot{\hat{e}})}{d \hat{e}} \dot{\hat{e}}, \quad \text { for } \tilde{k}^{\star}= \begin{cases}\tilde{k} & \text { if } \tilde{k} \geq 1 \\ 0 & \text { if } \tilde{k}<1\end{cases}
$$

with $\tilde{k}=\frac{1-\rho}{1-\rho_{0}(\hat{e} . \hat{e})}$. The factor $\tilde{k}^{\star}$ accounts for the instantaneous ${ }^{1-\hat{e}, \hat{e}}{ }^{*}$ change in strain rate. The homogenized void nucleation law in Eq. 7 is then expressed as:

$$
\dot{\bar{f}}_{\text {nucleation }}=V_{\mathrm{p}} \dot{\rho}
$$

$V_{\mathrm{p}}$ is a material parameter that relates the homogenized nucleated void volume fraction to the area fraction of cracked inclusions. The HCPD constitutive model developed here is used for simulating the material in level-0 computational subdomain $\Omega_{10}$.

\section{Level-1 Computational Subdomain $\Omega_{11}: A$ Swing Region}

The level-1 computational subdomain $\Omega_{11}$ is an intermediate, "swing" level that is used to assess whether a switchover is necessary from macroscopic analysis in level-0 subdomains to micromechanical analysis in level-2 subdomains. This subdomain is adaptively seeded in regions where macroscopic variables in level-0 simulations have locally high gradients of key variables. Level-1 subdomains utilize asymptotic expansion and SERVE periodicity ${ }^{3,4,11}$ to decouple the macroscopic and micro-SERVE problems for analyzing macroscopic and microscopic variables. Macroscopic finite-element analysis is done using the HCPD constitutive model, whereas micromechanical analysis of the SERVE, e.g., in Fig. 2c, is conducted with periodic boundary conditions and applied strain $\bar{e}$ from macroscopic analysis. Subsequently, adaptivity criteria (e.g., violation of boundary periodicity) are developed to decipher a transition from level-1 to level-2 elements.

\section{Computational Subdomain Level $2\left(\Omega_{12}\right)$ of Micromechanical Analyses}

Level-2 computational subdomains $\Omega_{12}$ of pure micromechanical analysis are regions with an image-based representation of microstructural heterogeneities as shown in Fig. 9. This subdomain adaptively replaces $\Omega_{11}$ in the multilevel computational domain. For an accurate and efficient micromechanical analysis, Ghosh developed the powerful VCFEM. ${ }^{11}$ Morphological nonuniformities in dispersions, shapes and sizes of micrographs are readily modeled by this method. The method has been extended to the locally enhanced VCFEM (LEVCFEM) in Ref. 35, to model stages of ductile fracture from particle fragmentation to matrix cracking due to void nucleation, growth and coalescence. In LE-VCFEM, the stress-based hybrid VCFEM formulation is adaptively altered in regions of localized plastic flow with finite deformation, displacementbased element formulation to accommodate strain softening. LE-VCFEM has been demonstrated to be very effective for simulating both rate-independent $^{35}$ and rate-dependent plasticity. ${ }^{22,36,37}$

The particulate phase in each Voronoi cell element is assumed to be isotropic, linear elastic. Instantaneous cracking and fragmentation commences when a size-dependent Weibull probability function $P_{\text {frag }}$ exceeds a critical value. 


$$
P_{\text {frag }}\left(v, \sigma_{I}^{c}\right)=1-\exp \left[-\frac{v}{v_{0}}\left(\frac{\sigma_{\mathrm{I}}^{c}}{\sigma_{\mathrm{w}}}\right)^{m}\right]
$$

where $m$ and $\sigma_{\mathrm{w}}$ are the Weibull modulus and characteristic strength, respectively; $v_{0}$ is a reference volume; $v$ is the inclusion size; and $\sigma_{I}^{c}$ is the maximum principal stress in the inclusion. The matrix phase in Ref. 36 is modeled as a rate-dependent elastic-viscoplastic porous material with void evolution, following the GTN model. The viscoplastic behavior of the porous ductile matrix is governed by the GTN yield function as:

$\phi^{v p}=\left(\frac{q}{\bar{\sigma}_{\mathrm{M}}}\right)^{2}+2 f^{*} q_{1} \cosh \left(-\frac{3 q_{2} p}{2 \bar{\sigma}_{\mathrm{M}}}\right)-\left(1+q_{3} f^{* 2}\right)=0$

$q=\sqrt{\frac{3}{2} \boldsymbol{\sigma}^{\prime}: \boldsymbol{\sigma}^{\prime}}$ and $p=-\frac{1}{3} \boldsymbol{\sigma}: \mathbf{I}$ are the Von-Mises equivalent stress and the hydrostatic pressure, respectively; $\sigma^{\prime}$ is the deviatoric stress; and $q_{1}, q_{2}$, and $q_{3}$ are void growth-related parameters. $f^{*}$ is a function of the void volume fraction $f$ that is expressed in Eq. 20. The subscript $\mathrm{M}$ is used to designate association with the pure matrix material without voids and $\bar{\sigma}_{\mathrm{M}}$ is the equivalent matrix stress. For the viscoplastic behavior, the overstress function $F_{\mathrm{M}}$ in Ref. 34 is expressed as:

$$
F_{\mathrm{M}}=\bar{\sigma}_{\mathrm{M}}-\sigma_{0}\left(W_{\mathrm{p}}\right)
$$

The plastic strain rate for the porous matrix is governed by the associated flow rule and is function of the overstress ${ }_{\mathrm{M}}\left(F_{\mathrm{M}}\right)$, expressed in Ref. 36 as:

$$
\dot{\epsilon}^{\mathbf{p}}=\dot{\lambda} \frac{\partial \phi^{v p}}{\partial \boldsymbol{\sigma}}=(1-f) \sqrt{\frac{2}{3}} \frac{\bar{\sigma}_{M}}{\boldsymbol{\sigma}: \frac{\partial^{v p}}{\partial \boldsymbol{\sigma}}} \gamma_{\mathrm{M}}\left(F_{\mathrm{M}}\right) \frac{\partial^{v p}}{\partial \boldsymbol{\sigma}}
$$

$\dot{\lambda}$ is a viscoplastic multiplier that is derived in terms of the matrix plastic strain rate $\dot{\epsilon}_{M}^{\mathbf{p}}$ and $\gamma$ is a temperature-dependent viscosity coefficient. A power law expression is chosen for $\mathrm{M}(F)=\left\langle F_{\mathrm{M}}\right\rangle^{p}$. A linear hardening law governs the evolution of the yield strength $\sigma_{0}$, expressed as:

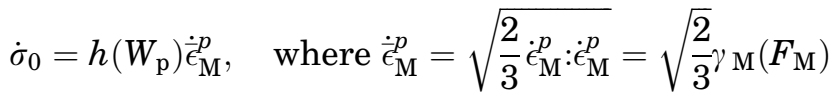

where $h\left(W_{\mathrm{p}}\right)$ is the instantaneous plastic modulus. The rate of evolution of the local void volume fraction $f$ is divided into growth and nucleation parts ${ }^{30,31}$ as:

$$
\begin{aligned}
\dot{f} & =\dot{f}_{\text {growth }}+\dot{f}_{\text {nucleation }} \text { where } \\
\dot{f}_{\text {growth }} & =(1-f) \dot{\epsilon}_{k h}^{p} \text { and } \\
\dot{f}_{\text {nucleation }} & =A\left(\bar{\epsilon}_{\mathrm{M}}^{p}\right) \dot{\bar{\epsilon}}_{\mathrm{M}}^{p}, \quad A\left(\bar{\epsilon}_{\mathrm{M}}^{p}\right)=\frac{f_{\mathrm{N}}}{s_{\mathrm{N}} \sqrt{2 \pi}} \\
& \exp \left[-\frac{1}{2}\left(\frac{\bar{\epsilon}_{\mathrm{M}}^{p}-\epsilon_{\mathrm{N}}}{s_{\mathrm{N}}}\right)^{2}\right]
\end{aligned}
$$

where $\epsilon_{\mathrm{N}}$ is the mean nucleation strain, $s_{\mathrm{N}}$ is its standard deviation, and $f_{\mathrm{N}}$ is the intensity of void nucleation. To avoid mesh sensitivity in LEVCFEM, a material length scale has been incorporated in Refs. 35 and 36 through a nonlocal model. The nonlocal growth rate of void volume fraction at a material point $\overline{\mathrm{x}}$ is given as:

$$
\dot{f}^{\text {nonlocal }}=\frac{1}{W(\overline{\mathbf{x}})} \int_{\Omega_{\mathrm{m}}} \dot{f}(\mathbf{x}) w(|\mathbf{x}-\overline{\mathbf{x}}|) \mathrm{d} \Omega
$$

where

$$
W(\overline{\mathbf{x}})=\int_{\Omega_{\mathrm{m}}} w(|\mathbf{x}-\overline{\mathbf{x}}|) \mathrm{d} \Omega \text { and } w(|\mathbf{x}|)=\left[\frac{1}{1+(|\mathbf{x}| / L)^{p}}\right]^{q}
$$

where $p=8, q=2$, and $L>0$ is a material characteristic length. The weighting function $w($ I $\mathbf{x} \mid)=1$ at $|\mathbf{x}|=0, w(|\mathbf{x}|)=0.25$ at $|\mathbf{x}|=L$ and $w(|\mathbf{x}|) \rightarrow 0 \forall|\mathbf{x}|>L$. Finally, an acceleration function $f^{*}$ is introduced in Eq. 13 to model the complete loss of material stress carrying capacity due to void coalescence $^{31}$ as:

$$
f^{*}= \begin{cases}f & f \leq f_{\mathrm{c}} \\ f_{\mathrm{c}}+\frac{f_{\mathrm{u}}^{*}-f_{\mathrm{c}}}{f_{\mathrm{f}}-f_{\mathrm{c}}}\left(f-f_{\mathrm{c}}\right) & f>f_{\mathrm{c}}\end{cases}
$$

$f_{\mathrm{c}}$ is the critical void volume fraction at which void coalescence first occurs and $f_{\mathrm{f}}$ is the value at final failure. As the void volume fraction $f \rightarrow f_{\mathrm{f}}$, the acceleration function $f^{*} \rightarrow f_{\mathrm{u}}^{*}=1 / q_{1}$. To avoid numerical difficulties, $f \rightarrow 0.95 f_{\mathrm{f}}$ is used in Eq. 20, at which $f$ is frozen, which implies local ductile material failure.

\section{Computational Subdomain Level tr $\left(\Omega_{t r}\right)$ for Interfacing Macroregions and Microregions}

The transition level-tr of sub-domain $\Omega_{\mathrm{tr}}$ is sandwiched between elements in level-0/level-1 and level-2 subdomains and facilitates a smooth transition of scales across the disparate element boundaries. Elements in $\Omega_{\text {tr }}$ are essentially level-2 elements that have compatibility and traction continuity constraints imposed on their interface with level-0/ level-1 elements. A relaxed, displacement-constraint method is incorporated in Refs. 11 and 20, where a weak form of the interface displacement continuity is enforced by using Lagrange multipliers.

\section{ADAPTIVITY AND COMPUTATIONAL LEVEL CHANGE CRITERIA}

The adaptive multilevel model needs robust criteria for level-0 mesh refinement, as well as for level transitions from macroscopic to microscopic scales with evolving deformation. Physics-based criteria are selected to drive the adaptation process as shown in Fig. 3. 


\section{Mesh Refinement for Level-o Elements}

Adaptive mesh refinement by h-adaptation or element subdivision ${ }^{38}$ in Fig. 3a, is conducted to reduce the discretization error associated with critical variables in level-0 elements. It also identifies regions of modeling error by zooming in on regions with evolving gradients. The adaptation criterion is formulated as: Refine element $e$ in $\Omega_{10}$ if the traction jump error across the element boundary satisfies the condition:

$$
E_{\mathrm{e}}^{t j} \geq C_{1} \max _{\mathrm{e}}\left(E_{\mathrm{e}}^{t j}\right) \text { where }\left(E_{\mathrm{e}}^{t j}\right)^{2}=\frac{\int_{\partial \Omega_{\mathrm{e}}}\left(\left[\left[T_{x}\right]\right]^{2}+\left[\left[T_{y}\right]\right]^{2}\right) d \partial \Omega}{\int_{\partial \Omega_{\mathrm{e}}} d \partial \Omega}
$$

The factor $C_{1}<1$ is chosen from numerical experiments. $T_{x}$ and $T_{y}$ are the boundary traction components in $x$ and $y$ directions, and [[?]] is the jump operator across the element boundary $\partial \Omega_{\mathrm{e}}$.

\section{Criteria for Switching from Level-0 to Level-1 Elements}

The transition from level-0 to level-1 elements is aimed at identifying regions of departure from homogenizability due to the intense local deformation and high gradients. For problems involving ductile deformation and damage, localization of macroscopic void volume fraction is an indicator of this departure. The transition of an element $e \in \Omega_{l 0}$ to $e \in \Omega_{l 1}$ is conditioned on the criterion:

$$
E_{\mathrm{e}}^{\mathrm{gdf}} f_{\mathrm{e}}^{\star} \geq C_{2} E_{\max }^{\mathrm{gdf}} f_{\max }^{\star}
$$

where $f^{\star}=\frac{\bar{f}-\bar{f}_{0}}{f_{0}}$ is the normalized void volume fraction with respect to the initial void volume fraction $\bar{f}_{0}$. In Eq. $22, E_{\mathrm{e}}^{\mathrm{gdf}}$ is the norm of the local gradient of $f^{\star}$ expressed as $E_{\mathrm{e}}^{\mathrm{gdf}}=\sqrt{\frac{\partial f_{\mathrm{e}}^{\star 2}}{\partial x}+\frac{\partial f_{\mathrm{e}}^{2}}{\partial y}}$. The quantities $f_{\max }^{\star}$ and $E_{\max }^{\text {gdf }}$ are the maximum values of all $f_{\mathrm{e}}^{\star}$ and $E_{\mathrm{e}}^{\mathrm{gdf}} . C_{2}<1$ is a prescribed factor determined from numerical experiments. The gradient of $f^{\star}$ is computed by first interpolating over a patch of elements by the super-convergent patch recovery (SPR) method in Ref. 38.

\section{Criteria for Switching from Level 1 to Level 2 Elements}

Transition from level 1 to level 2 is activated for elements that fail the macroscopic uniformity and RVE periodicity tests. Level 1 elements already correspond to those for which macroscopic nonuniformity has been established. Subsequently, departure from RVE periodicity is used as an indicator for a switch from level 1 to level 2 elements. The switching criterion is quantified as:

$$
T R_{\mathrm{e}}^{\mathrm{apt}}=\frac{\left\|\sum_{i=1}^{\mathrm{NSGPR}} \int_{\Gamma_{i}}\left(\left|t_{x}^{i+}+t_{x}^{i-}\right| \mathbf{i}+\left|t_{y}^{i+}+t_{y}^{i-}\right| \mathbf{j}\right) d \Gamma\right\|}{\max _{\mathrm{e}}\left\|\sum_{i=1}^{\mathrm{NSEG}} \int_{\Gamma_{i}}\left(\left|t_{x}^{i}\right| \mathbf{i}+\left|t_{y}^{i}\right| \mathbf{j}\right) d \Gamma\right\|}
$$

NSGPR is the number of boundary segment-pairs for each SERVE (shown in Fig. 2c) over which the stresses should be antiperiodic and NSEG is the total number of boundary segments on the SERVE. Tractions $t$ with superscripts + and - in the numerator correspond to those on the segment pairs with antiperiodicity conditions. The numerator is a measure of the residual traction violating the antiperiodicity condition. The denominator corresponds to the maximum value of the absolute sum of all traction measures in all the SERVEs of level 1 elements. $T R_{\mathrm{e}}^{\mathrm{apt}}$ provides a measure of the lack of antiperiodicity of boundary tractions, since $T R_{\mathrm{e}}^{\mathrm{apt}}=0$ if and only if the boundary tractions are anti-periodic. A level 1 element $e$ in $\Omega_{l 1}$ is switched to a level 2 microscopic element if:

$$
T R_{\mathrm{e}}^{\mathrm{apt}} \geq C_{3}
$$

where $C_{3}<1$ is a constant determined from numerical experiments. In addition, the level $0 /$ level 1 to level 2 switching criterion is also activated for elements undergoing significant damage according to the criterion:

$$
\rho_{\mathrm{e}} \geq \rho_{\text {crit }} \quad \forall e \in \Omega_{11}
$$

where $\rho_{\mathrm{e}}$ is the area fraction of cracked inclusions in the SERVE of level 1 element e. A value of $\rho_{\text {crit }}=0.1$

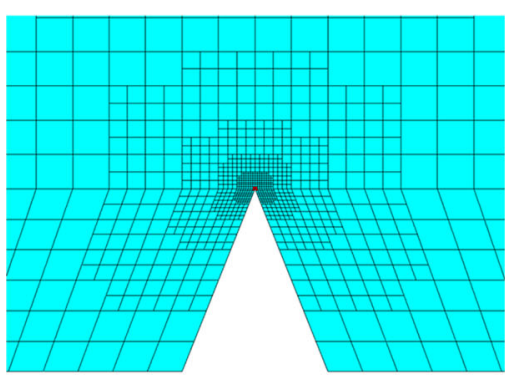

(a)

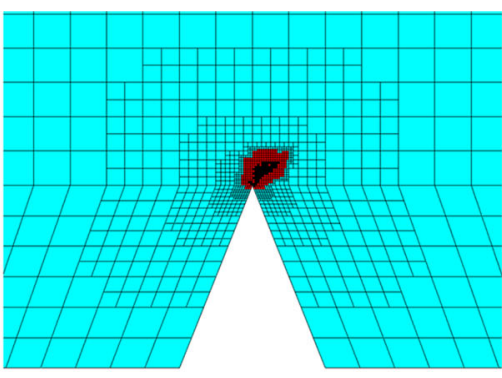

(b)

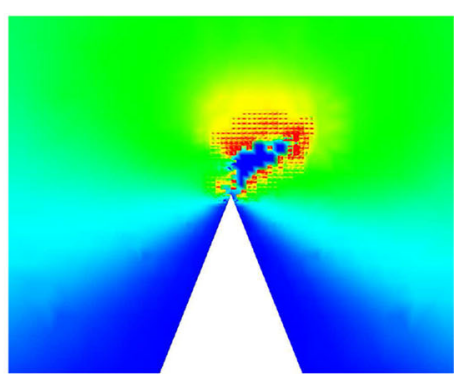

(c)

Fig. 3. Mesh and model adaptivity in multiscale analysis of a notched specimen loaded in tension (a) local mesh refinement by criterion (Eq. 21), Ref. 41 (b) evolution of levels [level 0 (turquoise), level 1 (blue), level 2/tr (red), and sealed elements (black)] using criteria (Eqs. 22, 24, and 25), and (c) contour plot of the microscopic stress component in the tension direction. 


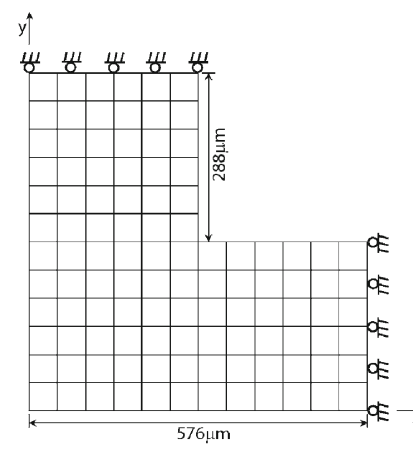

(a)

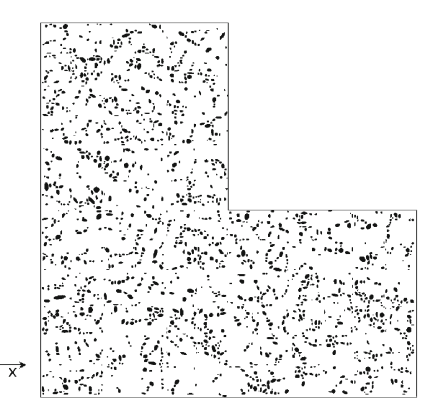

(b)

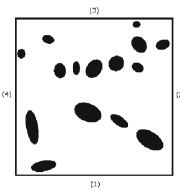

(c)

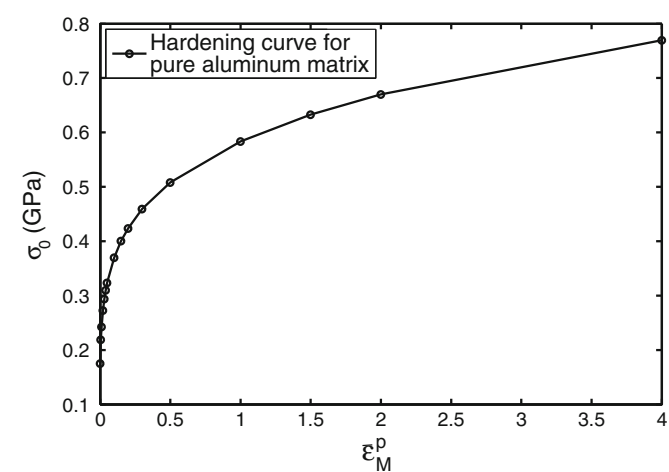

(d)

Fig. 4. (a) Geometry of the square plate with a center square hole, with symmetry boundary conditions; (b) underlying microstructure taken from a micrograph of W319 cast aluminum alloy; (c) SERVE (48 $\mu \mathrm{m} \times 48 \mu \mathrm{m})$; and (d) stress-strain behavior of the aluminum matrix used in LEVCFEM simulations.

significantly reduces the sensitivity of the solution to the parameter $C_{3}$ in Eq. 24. Figure $3 \mathrm{~b}$ and $\mathrm{c}$ shows the evolution of the model and stress evolution by the adaptive criteria.

\section{NUMERICAL STUDIES}

Numerical studies are undertaken to (I) calibrate the level-change criteria and validate the multiscale model, and (II) examine its capability for a realistic problem of multiscale ductile fracture.

\section{Validation of Multilevel Model and Level- Change Criteria}

The problem considered is a square plate with a square hole in its center with dimensions shown in Fig. 4a. Only one quarter of the plate is modeled, and appropriate symmetry boundary conditions are prescribed. In addition to the symmetry boundary conditions, a prescribed displacement $\Delta U_{A}$ is applied in the $x$ direction on the left edge $(x=0)$ of the plate. The bottom of the plate $(y=0)$ is free of any prescribed displacement, and no traction boundary conditions are applied. Constitutive parameters used for silicon particles and aluminum matrix in the micromechanical LE-VCFEM analysis are listed in Table I. The plastic hardening behavior of pure aluminum matrix (without voids or inclusions) is plotted in Fig. 4d.

Three types of solutions are considered in this example. First, the reference solution is obtained by solving a complete micromechanical analysis for the entire plate. In this simulation, all elements are level 2 containing the SERVE microstructure of Fig. 4c. In the next simulation, all elements are level 1 with an $\mathrm{FE}^{2}$ structure. Contour plots of $T R_{\mathrm{e}}^{\mathrm{apt}}$ are shown in Fig. 5a for an applied displacement of $U_{A}=-2.6 \mu \mathrm{m}$. A comparison of Fig. 5a with the evolution of microstructural deformation and damage in level 2 elements of the reference solution in Fig. $5 b$ demonstrates that $T R_{e}^{\text {apt }}$ successfully identifies regions of macroscopic nonuniformity. Subsequent tests are conducted with the concurrent multilevel (level $0 \rightarrow$ level $1 \rightarrow$ level 2 ) model to determine the values of $C_{3}$ and $\rho_{\text {crit }}$ in Eqs. 24 and 25, respectively. Figure 5c shows the contour plots of $\sigma_{x x}$ for multilevel analysis with $C_{3}=0.10$ and $\rho_{\text {crit }}=0.10$. Good agreement is found between the multilevel and micromechanical analysis results in Fig. 5b that validate the accuracy of the multiscale algorithm.

The multilevel model is solved for the boundary value problem of a cast aluminum alloy in Fig. 4 . The microstructure of the plate is shown in the micrograph of Fig. 4b. Figure $5 \mathrm{~d}$ and e shows the contour plots of the microscopic stress $\sigma_{x x}$ for an applied displacement $U_{A}=-4.32 \mu \mathrm{m}$ simulated by the adaptive concurrent multilevel model with $C_{3}=0.10$ and $\rho>0.10$ (Fig. 5d), and a micromechanical LEVCFEM model (Fig. 5e). The results agree very well, which confirms the effectiveness of the multilevel model in predicting ductile fracturing.

The results of another ductile fracture example with the same macroscopic L-shaped domain are shown in Fig. 6. However the microscopic SERVE is a unit cell comprising a single silicon inclusion of volume fraction $V_{\mathrm{f}}=10 \%$, aspect ratio $\alpha=2.0$, embedded in a square aluminum matrix region. Material parameters are the same as for the previous example with the following exceptions: $\sigma_{\mathrm{w}}=300 \mathrm{MPa}, \quad v_{0}=230.4 \mu \mathrm{m}^{2}, \quad P_{\text {frag }}^{c r}=95 \%$, and $\epsilon_{N}=0.10$. The domain is subjected to prescribed boundary conditions: $U_{x}=-\Delta U$ at $y=576 \mu \mathrm{m}$, $U_{x}=0$ at $x=576 \mu \mathrm{m}$, and $U_{y}=0$ at $(x$, $y)=(576 \mu \mathrm{m}, 0)$, where $\Delta U$ is incremented until fracture occurs. Figure $6 \mathrm{~b}$ shows the evolved multilevel mesh at the end of the loading sequence. At failure, the level 2 element is replaced by a failed macroscopic element (black elements in Fig. 6b). Figure 6 a shows a comparison of the total reaction force per unit length at the edge $x=576 \mu \mathrm{m}$ as a function of the applied displacement $U_{x}$ by the multilevel and micromechanical simulations. A very good match is obtained between the two models. Each drop in the multilevel 
model results from failure of the underlying level 2 microstructure. Contour plots of equivalent plastic strain $\bar{\epsilon}_{\mathrm{M}}^{p}$ by the two methods at final fracture in the Fig. 6c and $d$ show concurrence in predicting the ductile crack path. This example further demonstrates the accuracy of the multilevel model for solving the multiscale ductile fracture problems.

\section{Multiscale Simulation of Ductile Failure in a Cast Aluminum Bar}

The multilevel model is now applied for multiscale ductile fracture analysis of an $\mathrm{Al}-\mathrm{Si}-\mathrm{Mg}$ cast aluminum alloy W319 rectangular bar, for which the micrograph is shown in Fig. 2b. It comprises an agehardened ductile aluminum matrix, strengthened

Table I. (a) Inclusion elastic and cracking properties and (b) aluminum matrix elastic, plastic, and void evolution properties used in LE-VCFEM simulations

(a)

\begin{tabular}{llllll}
\hline $\boldsymbol{E}(\mathbf{G P a})$ & $\frac{\boldsymbol{v}}{165}$ & $\frac{\boldsymbol{\sigma}_{\mathrm{w}}(\mathbf{M P a})}{680}$ & $\frac{\boldsymbol{m}}{2.4}$ & $\frac{\boldsymbol{v}_{\mathbf{0}}\left(\mu \mathbf{m}^{2}\right)}{8.29}$ & $\frac{\boldsymbol{P}_{\text {frag }}^{\mathrm{cr}}(\%)}{55}$ \\
\hline
\end{tabular}

(b)

\begin{tabular}{lllllllllll}
\hline $\boldsymbol{E}$ (GPa) & $\frac{\boldsymbol{v}}{70}$ & $\frac{\gamma_{\mathbf{0}}\left(\mathbf{G P a}^{-\mathbf{1}} \mathbf{s}^{-\mathbf{1}}\right)}{8.3}$ & $\frac{\boldsymbol{p}}{1}$ & $\frac{\boldsymbol{f}_{\mathbf{o}}}{0.01}$ & $\frac{\boldsymbol{f}_{\mathbf{c}}}{0.15}$ & $\frac{\boldsymbol{f}_{\mathbf{f}}}{0.25}$ & $\frac{\epsilon_{\mathbf{N}}}{0.2}$ & $\frac{\boldsymbol{s}_{\mathbf{N}}}{0.075}$ & $\frac{\boldsymbol{f}_{\mathbf{N}}}{0.08}$ \\
\hline
\end{tabular}

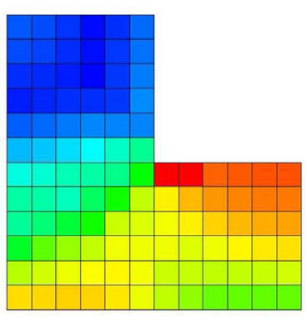

(a)

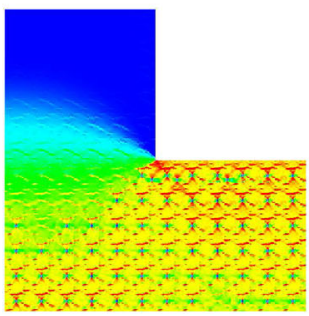

(b)

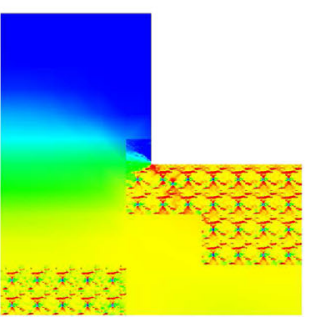

(c)

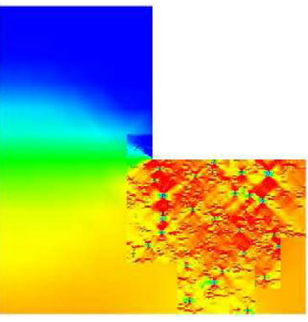

(d)

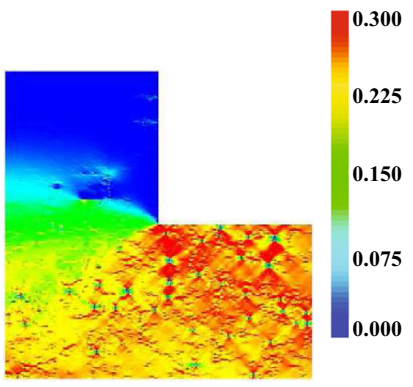

(e)

Fig. 5. Contour plots of: (a) $T R_{\mathrm{e}}^{\mathrm{apt}}$ in a macroscopic level 1 simulation indicating departure from traction antiperiodicity, (b) $\sigma x x(\mathrm{GPa})$ for a fully microscopic level 2 simulation, and (c) $\sigma x x(\mathrm{GPa})$ at multilevels of the concurrent multiscale model with $\mathrm{C} 3=0.10$ and $\rho>0.10$, all for $\mathrm{UA}=-2.6 \mu \mathrm{m}$. Micromechanical stress $\sigma x x(\mathrm{GPa})$ contours for the complex microstructure by (d) adaptive multilevel simulation and (e) fully micromechanical simulation.

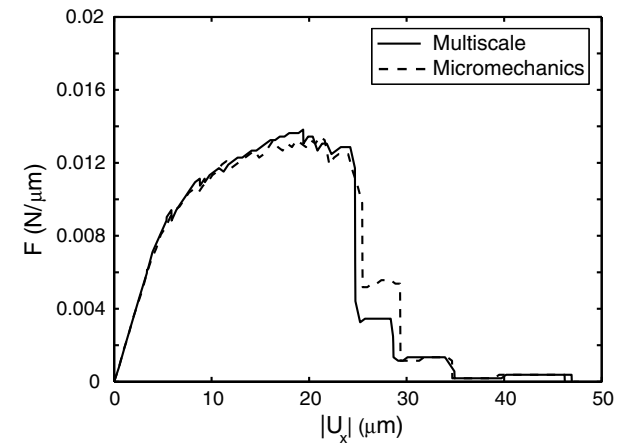

(a)

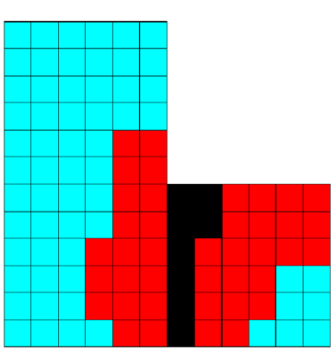

(b)

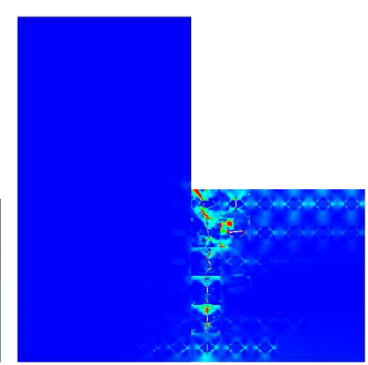

(c)

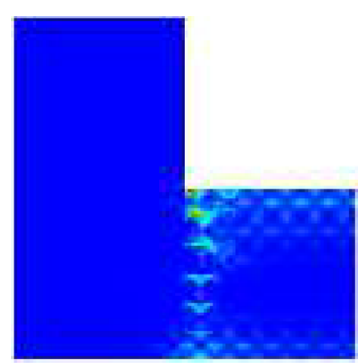

(d)

Fig. 6. (a) Comparison of the total reaction force per unit length $F / L$ at $x=576 \mu \mathrm{m}$ as a function of applied displacement $U x$ by multilevel and micromechanical analyses. (b) Evolved adaptive multilevel mesh at the end of simulation [level 0 (turquoise), level 1 (blue), level 2 and level tr (red), and failed elements (black)]. Contour plot showing the equivalent plastic strain and final crack path by (c) multilevel model and (d) micromechanical model. 
by $\mathrm{Mg} / \mathrm{Si}$ and a dispersion of brittle Si particulates. Dimensions of the rectangular computational domain are: $384 \mu \mathrm{m} \times 1,536 \mu \mathrm{m}$. A high-resolution microstructure of the alloy is mapped on the rectangular specimen following the procedures in Ref. 25 .

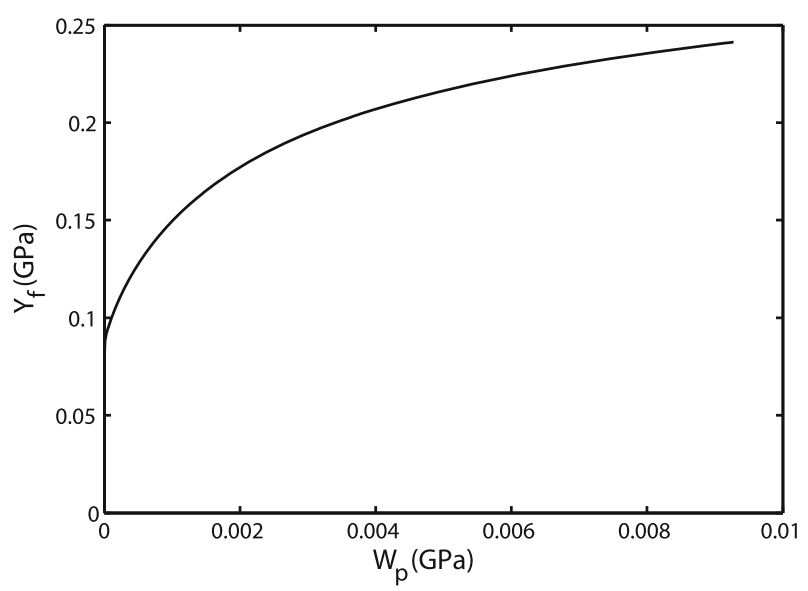

(a)

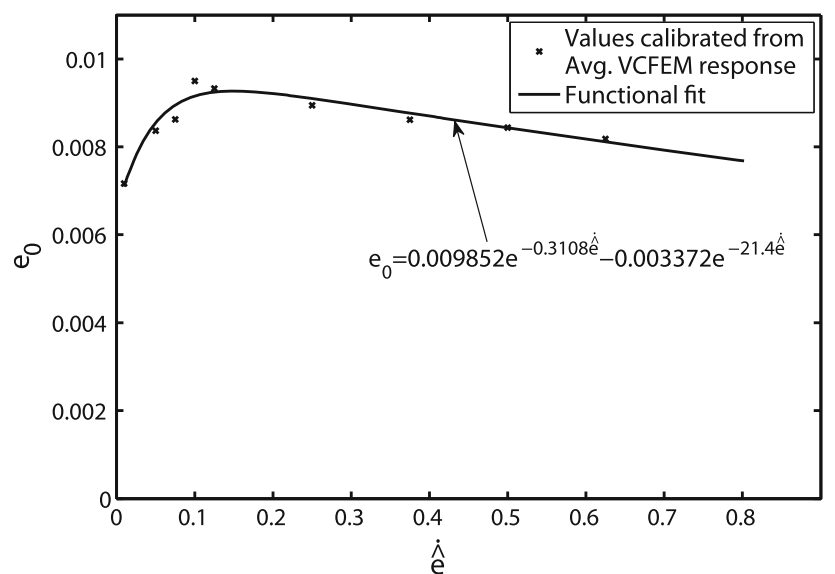

(b)

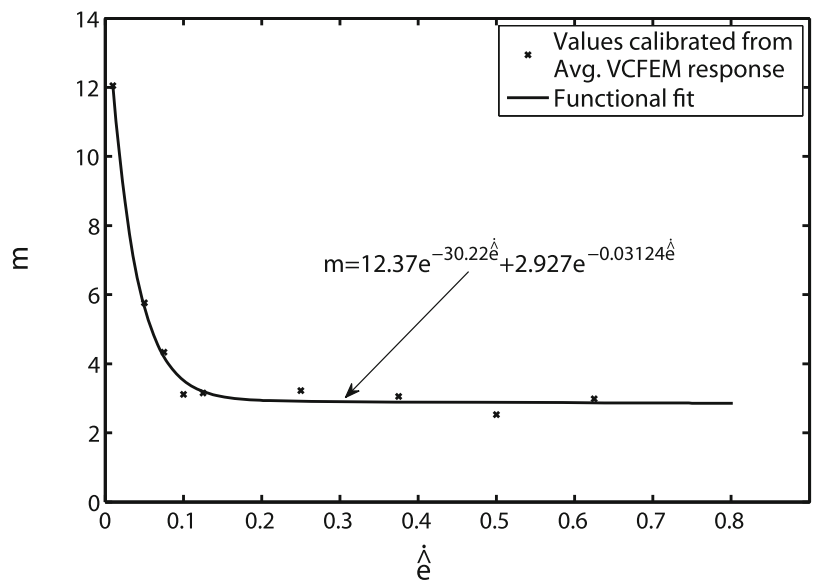

(c)

Fig. 7. Evolution of (a) yield stress in shear $Y_{f}$ with plastic work, and (b) $e_{0}$ and (c) $m$ with local strain rate $\dot{\hat{e}}$.
The multilevel model is initiated with a level-0 computational domain $\Omega_{10}$ for the entire bar and then discretized into 256 elements as shown in Fig. 8. The HCPD model developed uses a SERVE of size $48 \mu \mathrm{m}$ shown in Fig. 2c. The parameters for the rate-dependent HCPD model were calibrated in Ref. 28.

The evolution of yield strength $Y_{\mathrm{f}}\left(W_{\mathrm{p}}\right)$ in Eq. 5) and parameters $e_{0}$ and $m$ in Eq. 8 are shown in Fig. 7. The homogenized viscoplastic parameters $\Gamma_{0}=6.13 \mathrm{GPa}^{-1} \mathrm{~s}^{-1}$ and $P=1$, and the parameters in Eq. 3 are $Q_{1}=1.89$ and $Q_{2}=1.01$.

The tension specimen is simulated for uniaxial tension using the boundary conditions: $U_{y}=\Delta U$ at $y=1536 \mu \mathrm{m}, \quad U y=0 \quad$ at $y=0, \quad\left(U_{x}=0 \quad\right.$ at $(x$, $y)=(0,0)$. In Refs. 36 and 37, it was established that regions of high local inclusion volume fraction and clustering have low ductility. A function $\tilde{f}$ of the inclusion volume fraction $V_{\mathrm{f}}$ and cluster contour index $l$ is given as:

$$
\tilde{f}=\frac{l}{0.929-1.83 V_{\mathrm{f}}}
$$

is a very good indicator of local ductility. Prior to the multiscale analysis, $\tilde{f}$ is computed for the local microstructure of each of the 256 level- 0 elements, and six critical elements (regions) are identified as hot spots for premature nucleation of ductile cracks. Correspondingly, in a concurrent setting, these are modeled as level 2 elements from the start. The remainder of all elements in the initial multilevel mesh are level 0 as shown in Fig. 8a. With increasing deformation, nonuniformities arise in the microscopic subdomains $\Omega_{12}$ due to cracking of silicon inclusions and plastic deformation of the aluminum matrix, thus altering the initial homogeneity of the macroscale stress and strain fields. This leads to high gradients in the void volume fraction $\bar{f}$ that switch the surrounding level 0 elements to level 1 elements, and subsequently precipitates level 2 elements in these regions. This adaptive transition of macroscopic to microscopic elements, shown in Fig. 8, continues with the evolution of damage primarily in a direction perpendicular to the applied load. These microscopic elements in $\Omega_{12}$ coalesce with the evolving ductile deformation and damage. The contour plot of microscopic $\sigma_{y y}$ at the onset of local failure is given in Fig. 8h, and an image of the underlying microstructure is shown in Fig. 8g. The contour plots of microscopic void volume fraction and equivalent plastic strain for the newly failed elements are shown in Fig. 9. These plots clearly demonstrate that a dominant ductile crack has propagated through the level 2 microstructure.

\section{DUAL-STAGE NESTED HOMOGENIZATION FOR RATE-DEPENDENT HCP MODEL OF DENDRITIC CAST ALUMINUM ALLOYS}

This section discusses the development of a special homogenization process that is very effective for 


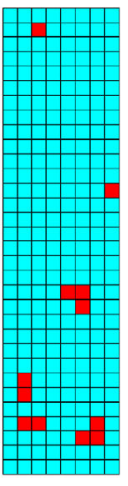

(a)

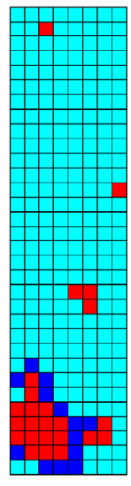

(b)

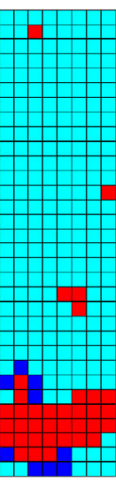

(c)

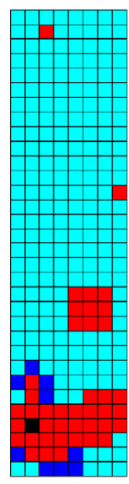

(d)

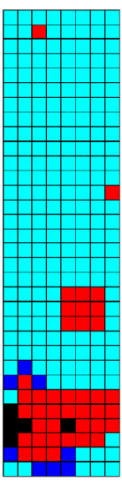

(e)

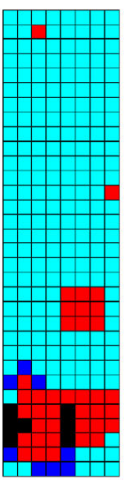

(f)

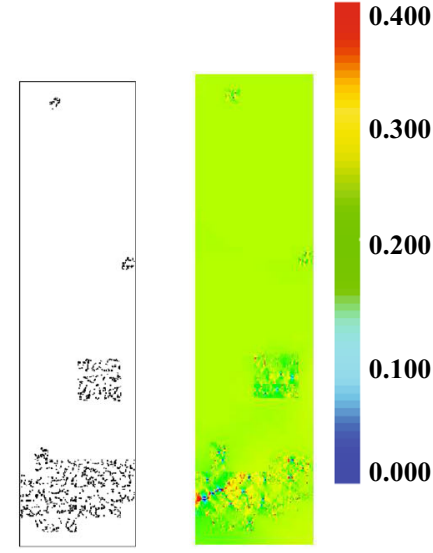

(g)

(h)

Fig. 8. Evolution of the adaptive multilevel mesh for the multiscale analysis of a rectangular specimen loaded in tension, at: (a) $U=0$, (b) $\mathrm{U}=7.8 \mu \mathrm{m}$, (c) $\mathrm{U}=10.3 \mu \mathrm{m}$, (d) $U=13.2 \mu \mathrm{m}$, (e) $U=13.5 \mu \mathrm{m}$, (f) $U=13.7 \mu \mathrm{m}$ [Legend: level 0 (turquoise), level 1 (blue), level 2 and level tr (red), and sealed elements (black)]. (g) Underlying microstructure of the level 2 elements in the multilevel mesh and (h) contour plot of microscopic stress $\sigma y y(\mathrm{GPa})$ for the computational domain of the tensile specimen at an applied displacement Uy $=13.0 \mu \mathrm{m}$.

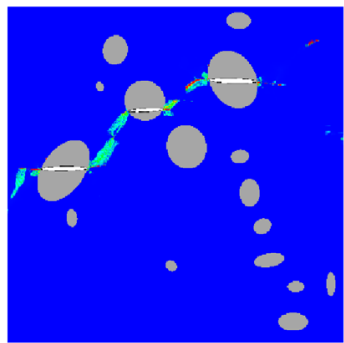

(a)

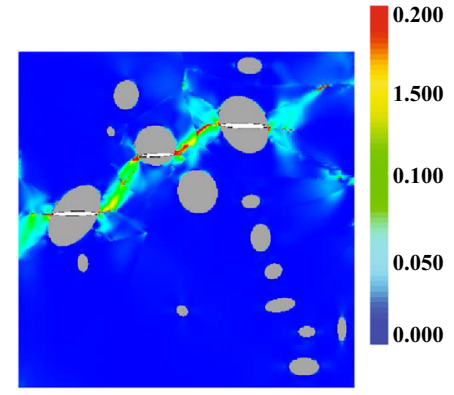

(b)
Fig. 9. Contour plots showing the final crack path at complete failure of the first level 2 element during the multiscale simulation: (a) void volume fraction $f$ and (b) equivalent plastic strain $\bar{\epsilon}_{\mathrm{M}}^{p}$.

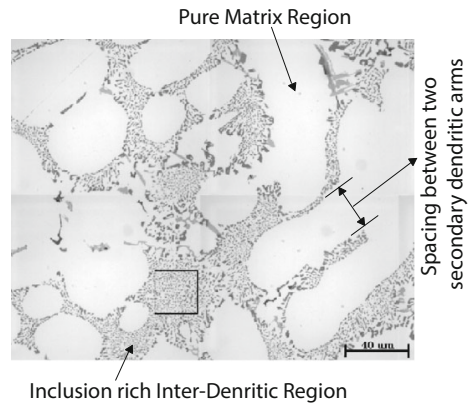

(a)

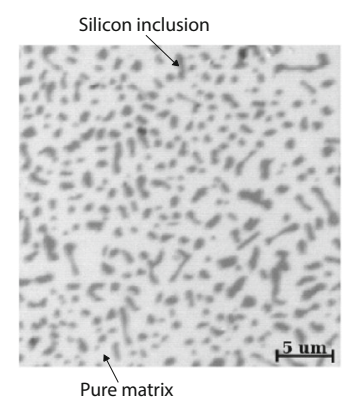

(b)
Fig. 10. (a) Micrograph of a cast aluminum alloy AS7GU $(120 \mu \mathrm{m} \times 96 \mu \mathrm{m})$ and (b) blow-up of the designated region in (a) $(28.4 \mu \mathrm{m} \times 28.4 \mu \mathrm{m})$.

microstructures characterized by disparate distribution of heterogeneities, as shown for a cast aluminum alloy with large SDAS in Fig. 10. The distribution of silicon inclusions along the dendrite cell boundaries delineates two distinct material phases viz. (I) a heterogeneity-free pure aluminum matrix and (II) an interdendritic phase (IDP) in which silicon inclusions are dispersed in the aluminum matrix. The spatial distribution of inclusions is characterized by the secondary dendrite arm spacing, which for this microstructure is $\sim 30 \mu \mathrm{m}$. Traditional single-step homogenization methods are not suitable for this type of microstructure due to the discrepancy of scales. A single RVE for the microstructure containing dendrites and IDPs may lead to dimensions exceeding the available micrograph and contain a large number of heterogeneities. This makes their simulation computationally prohibitive.

To circumvent this limitation, a dual-stage nested homogenization method was developed in Ref. 27 for developing microstructure-based HCP models. It takes advantage of the inherent scales of heterogeneity in the microstructure and eliminates the need for micromechanical analyses of a large, single RVE for the entire microstructure. Instead, it identifies multiple SERVEs, corresponding to the scales of microstructural heterogeneity. Two scales of heterogeneity are associated with the microstructure in Fig. 10. They are: (I) the length scale ( $m s-1)$ of intraIDP $\mathrm{Si}$ inclusions in the aluminum matrix in Fig. $10 \mathrm{~b}$ and (II) the length scale $(m s-2)$ of dendrite cells in Fig. 10a associated with the larger scale of pure aluminum dendrites and the IDPs. The nested homogenization process to determine the macroscopic rate-dependent, anisotropic HCP model is performed in multiple stages for SERVEs at each scale. The process enables the evaluation not only of the overall homogenized constitutive model parameters from limited experimental data but also of the constituent material parameters e.g., for interdendritic phase and pure aluminum.

Two methods are invoked sequentially in the nested homogenization model of Fig. 12. 


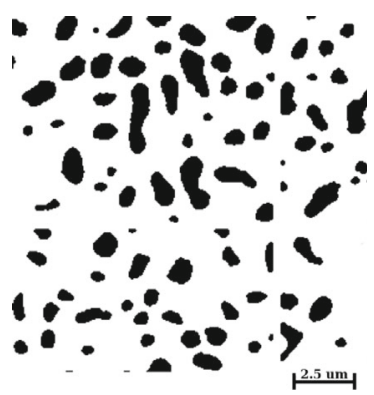

(a)

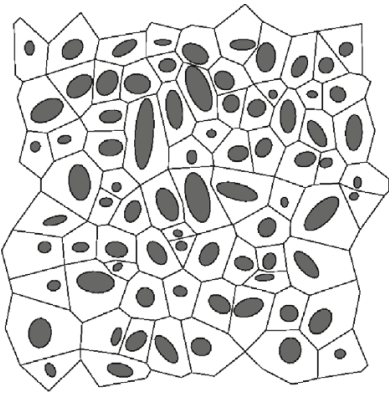

(b)

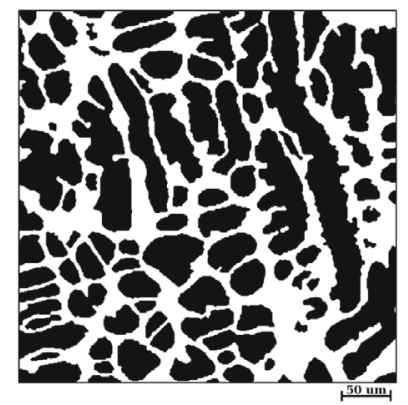

(c)

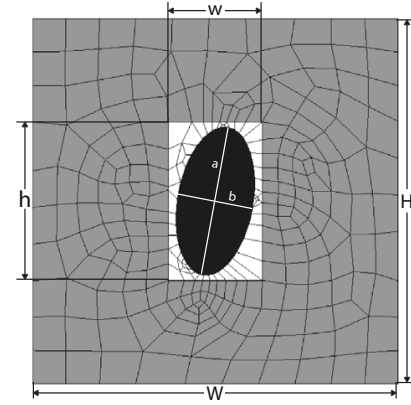

(d)

Fig. 11. (a) Micrograph of the microstructural interdendritic IDP domain (scale $2.5 \mu \mathrm{m}$ ), (b) a VCFEM model of the SERVE in the IDP domain for asymptotic homogenization, (c) simulated micrograph of Fig. 10a (scale $50 \mu \mathrm{m}$ ) showing the distribution of pure aluminum matrix (black) and interdendritic (white) phases, (d) FEM model for the self-consistent homogenization: pure matrix (black), interdendritic phase (white), and homogenized overall material (gray).

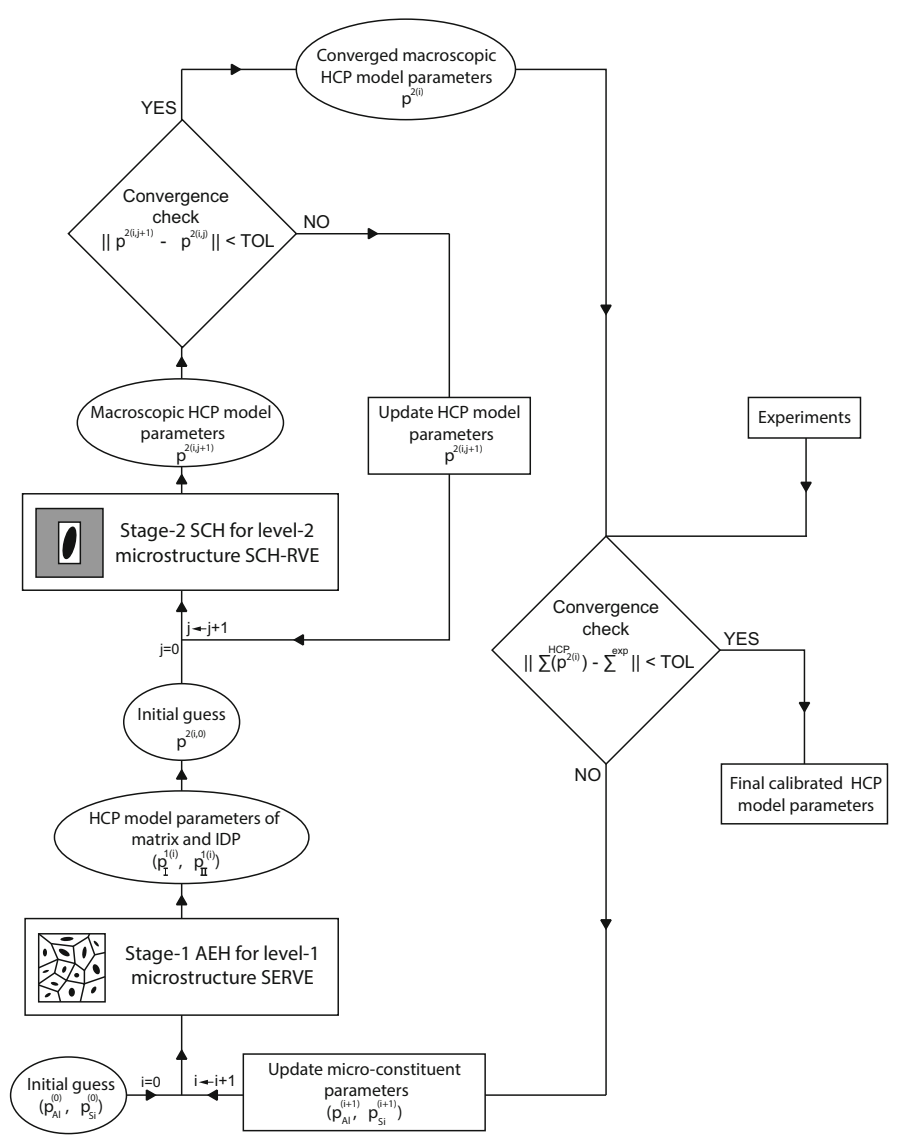

(a)

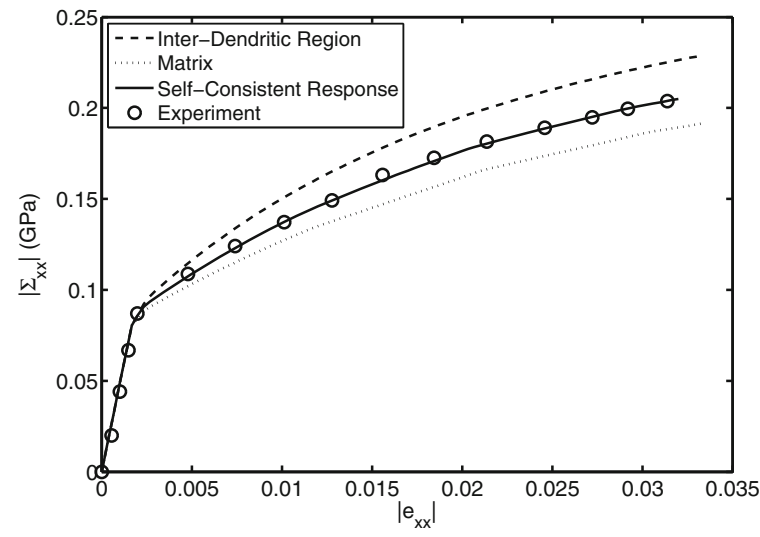

(b)

Fig. 12. (a) Schematic flow chart of the nested two-stage homogenization method for large SDAS cast aluminum alloys. (b) Comparison of the self-consistent responses of the pure matrix, interdendritic phase, and the overall cast aluminum alloy in compression.

\section{Asymptotic Expansion-Based Homogenization (AEH) of $m s-1$ Intra-IDP SERVEs}

This stage develops an effective HCP constitutive model for the IDP region shown in Fig. 10b by using $\mathrm{AEH}{ }^{4,26,28}$ The microscale problem involves identification of a SERVE and subsequent micromechanical analyses with LE-VCFEM). ${ }^{35,36}$ Parameters of the HCP model are calibrated from homogenization of evolving variables in the SERVE of Fig. 11. 


\section{Self-Consistent Homogenization (SCH) of $\mathrm{ms}-2$ Microstructure Containing Dendrites and IDPs}

This stage generates an effective HCP constitutive model for the overall alloy microstructure, containing dendritic cells of aluminum matrix and inclusion-rich IDP regions shown in Fig. 11c, by a self-consistent scheme that equilibrates a representative dendrite cell and its neighboring IDP in the overall microstructure. Self-consistent models assume no spatial correlation at the microstructural scale and admit complete scale separation. Each heterogeneity views the rest of the medium as a homogeneous material in Fig. 11d, having yet undetermined macroscopic properties. ${ }^{39}$

A schematic of the nested dual-stage homogenization scheme, implementing $\mathrm{AEH}$ and $\mathrm{SCH}$ is shown in Fig. 12a. The homogenization scheme is applied to calibrate material parameters and model the mechanical response of the cast aluminum alloy of Fig. 10 having a SDAS of $30 \mu \mathrm{m}$. The experimental data for compression tests are generated ${ }^{40}$ and the stress-strain plot is shown in Fig. 12b. The figure also compares the respective responses of the pure matrix and interdendritic phases with the overall response of the cast alloy in compression. An excellent match is observed between the experimental data and the simulated results. A number of other satisfactory validation tests were conducted in Ref. 27.

\section{CONCLUSION}

This article assimilates a variety of complementary ingredients to create an adaptive hierarchicalconcurrent multiscale modeling framework necessary for modeling ductile fracture in heterogeneous metals and alloys. Two relevant topics are reviewed in this article. The first topic discusses major ingredients of the adaptive hierarchical-concurrent multiscale framework. The adaptive capability enables this top-down coupling for the evolutionary problems in an automatic fashion. Numerical simulations show that damage initiation and growth can be effectively captured by the model without the introduction of any artificial discontinuity or defect. The multilevel adaptive model imparts the necessary efficiency that is essential to conduct such large-scale computations. Few models in the literature represent the concurrent setting to manifest localization of deformation and damage within the microstructure. An unique feature is the incorporation of detailed microstructural information acquired from micrographs. While the framework in this article is developed for alloys containing a distributions of precipitates, the methods are easily adapted to the analysis and design of a larger class of metal-matrix composites with micron-size particles/fibers and interparticle spacing.

The second topic introduces a novel two-stage homogenization scheme to develop homogenized constitutive models for microstructures exhibiting large-scale discrepancies, e.g., in cast aluminum alloys with SDAS. The method is based on the inherent scales of inhomogeneity in the microstructure, thus overcoming limitations associated with one-step homogenization techniques. It enables evaluation not only of the overall homogenized model parameters of the cast alloy from experimental data but also of constituents, such as interdendritic phase, aluminum matrix, and $\mathrm{Si}$ inclusions. The multiscale evaluation of multimaterial properties is a unique feature that can provide a significant advantage with limited experimental data at fewer scales.

\section{ACKNOWLEDGEMENTS}

The author acknowledges the contributions of many of his past graduate students, especially D. Paquet, P. Dondeti, J. Bai, and H. Chao, on various parts of this work. This work was supported by the National Science Foundation NSF Division of Civil and Mechanical Systems Division through the GOALI Grant No. CMS-0308666 (Program Directors: Dr. M. Dunn and Dr. T. Siegmund) and by the Army Research Office through Grant No. DAAD1902-1-0428 (Program Director: Dr. R. Anthenien). This sponsorship is gratefully acknowledged. The author is grateful to colleagues at Ford Research Laboratory for micrographs and samples.

\section{REFERENCES}

1. Q.G. Wang, C.H. Caceres, and J.R. Griffiths, Metall. Mater. Trans. A 34, 2901 (2003).

2. M. Guedes and N. Kikuchi, Comput. Methods Appl. Mech. Eng. 83, 143 (1990).

3. S. Ghosh, K. Lee, and S. Moorthy, Int. J. Solids Struct. 27, 143 (1995)

4. S. Ghosh, K. Lee, and S. Moorthy, Comput. Methods Appl. Mech. Eng. 132, 63 (1996).

5. K. Lee and S. Ghosh, Comput. Mater. Sci. 7, 131 (1996).

6. T.I. Zohdi, J.T. Oden, and G.J. Rodin, Comput. Meth. Appl. Mech. Eng. 172, 3 (1999).

7. F. Feyel and J.-L. Chaboche, Comput. Meth. Appl. Mech. Eng. 183, 309 (2000).

8. K. Terada and N. Kikuchi, Comput. Meth. Appl. Mech. Eng. 190, 5427 (2001).

9. C. Miehe and A. Koch, Arch. Appl. Mech. 72, 300 (2002).

10. H. Moulinec and P. Suquet, Int. J. Numer. Meth. Eng. 52, 139 (2001).

11. S. Ghosh, Micromechanical Analysis and Multi-scale Modeling using the Voronoi Cell Finite Element Method (Boca Raton, FL: CRC Press, Taylor \& Francis, 2011).

12. M.G.D. Geers, V.G. Kouznetsova, and W.A.M. Brekelmans, J. Comput. Appl. Math. 234, 2175 (2010).

13. D.J. Luscher, D.L. McDowell, and C.A. Bronkhorst, Int. J. Plastic. 26, 1248 (2010).

14. F.J. Vernerey, W.K. Liu, and G. Olson, J. Mech. Phys. Solids 56,1320 (2008).

15. J. Fish and S. Kuznetsov, Int. J. Numer. Meth. Eng. 84, 774 (2010).

16. T. Belytschko, S. Loehnert, and J.-H. Song, Int. J. Numer. Meth. Eng. 73, 869 (2008).

17. P. Raghavan, S. Moorthy, S. Ghosh, and N.J. Pagano, Compos. Sci. Technol. 61, 1017 (2001).

18. P. Raghavan and S. Ghosh, Comput. Meth. Appl. Mech. Eng. 193, 497 (2004). 
19. K. Lee, S. Moorthy, and S. Ghosh, Comput. Meth. Appl. Mech. Eng. 172, 175 (1999).

20. S. Ghosh, J. Bai, and P. Raghavan, Mech. Mater. 39, 241 (2007).

21. S. Ghosh, K. Lee, and P. Raghavan, Int. J. Solids Struct. 38, 2335 (2001).

22. S. Ghosh and D. Paquet, Mech. Mater. 65, 12 (2013).

23. S. Ghosh, Multiscale Modeling of Complex Materials, CISM Lectures 556, eds. Y. Sadowski and P. Trovalusci (New York: Springer, 2014).

24. D.M. Valiveti and S. Ghosh, Int. J. Num. Meth. Eng. 69, 1717 (2007).

25. S. Ghosh, D.M. Valiveti, S.J. Harris, and J. Boileau, Model. Simul. Mater. Sci. Eng. 14, 1363 (2006).

26. S. Ghosh, J. Bai, and D. Paquet, J. Mech. Phys. Solid. 57, 1017 (2009).

27. D. Paquet, P. Dondeti, and S. Ghosh, Int. J. Plastic. 27, 1677 (2011).

28. P. Dondeti, D. Paquet, and S. Ghosh, Eng. Fract. Mech. 89, 75 (2012).
29. A.L. Gurson, J. Eng. Mater. Tech. 99, 2 (1977).

30. C.C. Chu and A. Needleman, J. Eng. Mater. Tech. 102, 249 (1980).

31. V. Tvergaard and A. Needleman, Acta Metall. 32, 157 (1984).

32. R. Hill, Proc. R. Soc. A Math. Phys. 326, 131 (1972),

33. R. Hill, Proc. R. Soc. A Math. Phys. 193, 281 (1948).

34. P. Perzyna, Adv. Appl. Mech. 9, 243 (1966).

35. C. Hu and S. Ghosh, Int. J. Numer. Meth. Eng. 76, 1955 (2008).

36. D. Paquet and S. Ghosh, Eng. Fract. Mech. 78, 205 (2011).

37. D. Paquet and S. Ghosh, Eng. Fract. Mech. 78, 226 (2011).

38. D. Paquet (Ph.D. thesis, The Ohio State University, 2011).

39. S. Nemat-Nasser and M. Hori, Micromechanics: Overall Properties of Heterogeneous Materials, Vol. 207 (New York: Elsevier, 1999).

40. K. Chisaka (B.S. thesis, The Ohio State University, 2009).

41. O.C. Zienkiewicz and J.Z. Zhu, Int. J. Numer. Meth. Eng. 33, 1331 (1992). 